J. Beerten, S. D'Arco and J.A. Suul, “Cable model order reduction for HVDC systems interoperability analysis," Proc. IET International Conference on AC and DC Power Transmission ACDC 2015, 11th ed., Birmingham, UK, Feb. 10-12, 2015, 10 pages.

Digital Object Identifier: 10.1049/cp.2015.0039

URL (IET Digital Library):

http://digital-library.theiet.org/content/conferences/10.1049/cp.2015.0039

URL (IEEE Xplore Digital Library):

http://ieeexplore.ieee.org/xpl/articleDetails.jsp?arnumber=7140534

(C) 2015 IET. This paper is a postprint of a paper submitted to and accepted for publication in Proc. IET International Conference on AC and DC Power Transmission 2015 and is subject to Institution of Engineering and Technology Copyright. The copy of record is available at IET Digital Library. 


\title{
Cable Model Order Reduction for HVDC Systems Interoperability Analysis
}

\author{
J. Beerten ${ }^{*, \dagger}$, S. D’Arco ${ }^{\ddagger}$, J.A. Suul ${ }^{\dagger, \ddagger}$ \\ * Department of Electrical Engineering (ESAT), Division ELECTA \& Energyville, University of Leuven (KU Leuven), Belgium \\ $\dagger$ Dept. Electric Power Engineering, Norwegian University of Science and Technology (NTNU), Trondheim, Norway \\ $\ddagger$ SINTEF Energy Research, Trondheim, Norway
}

Keywords: State-space modelling, VSC HVDC, cable modelling, converter interoperability, small-signal stability.

\begin{abstract}
In order to predict the stability of cable-based offshore Voltage Source Converter High Voltage Direct Current (VSC HVDC) systems, an accurate cable model is needed to represent the characteristics of the transmission system. This paper presents a model consisting of cascaded pi-sections with multiple longitudinal parallel branches, allowing for a detailed representation in the frequency domain. Furthermore, a cable model order reduction for system interoperability studies is proposed based on the identification of interacting modes. Simulation results demonstrate how a classical cascaded pi-model is unable to accurately represent the interacting modes and show how the state-space representation of the proposed cable model can be reduced whilst preserving an accurate representation of the converter interactions.
\end{abstract}

\section{Introduction}

As a consequence of the expected increase in offshore wind farm development at long distances from shore, the electric power industry is showing a growing interest in High Voltage Direct Current (HVDC) transmission based on Voltage Source Converter (VSC) technology in multi-terminal and even meshed grid configurations. Such future large-scale HVDC grids are expected to be developed step-by-step, and will likely be multi-vendor systems with potentially different converter technologies operating in the same grid. Interoperability and the prediction of possible adverse interactions between the converter terminals are therefore critical aspects of concerns. This scenario creates open research questions on system modelling techniques, especially in the perspective of reduced order models for stability assessment of converters in offshore VSC-based HVDC grids. Detailed component models exist for electromagnetic transient (EMT) simulations, but these can generally only be used for case-specific time domain simulations.

Until now, the focus for control interactions has traditionally been on the stability of the VSC HVDC system with respect to the $\mathrm{AC}$ side. It has therefore been common practice to directly reduce the order of the DC system model by straightforwardly representing the cables as either pi-equivalent circuits or even a resistive network. Although the classical pi-equivalent representation yields simple analytical expressions, it is to a large extent an open question whether it suffices for developing statespace models to address all potential unintended interactions in HVDC grids. It has also recently been confirmed by the results in [1] that the classical pi-equivalent representation could give false indications on the stability of converters in an HVDC system. Since detailed frequency-dependent cable models for EMT simulations can hardly be translated into state-space representations, a systematic approach is missing. This paper aims at addressing this lacuna by developing a detailed cable model for small-signal studies and by providing an approach towards reduced order modelling for HVDC grids, based on an accurate representation of the cable characteristics.

The model order reduction technique proposed in this paper takes the frequency response characteristics of the HVDC grid into account by including a frequency-dependent model of the DC cable. First, section 2 analyses the main drawbacks of commonly used models for small-signal control studies by providing a systematic introduction to cable modelling for a state-space representation. Next, section 3 presents an alternative cable model, based on the modelling approach presented in [2] and applied for a state-space representation of transmission lines in [3]. The model can accurately represent the frequency-dependency of the cable by applying vector fitting, as presented in [4], for the series elements. Section 4 discusses the modelling of the HVDC system in a state-space representation. Section 5 introduces the proposed model reduction. Finally, section 6 presents the simulation results.

\section{Cable modelling}

\subsection{Steady-state cable model}

For converter control interactions studies, the model of HVDC cables can be reduced by means of a Kron reduction. This assumes an ideal grounding for both the armour and sheath conductors, namely that they are at ground potential along the entire length of the cable. In reality, the cable sheaths are usually grounded at both ends. The reduction therefore only applies 
when the voltages in armour and sheath remain small compared to the ones on the conductor [5], which is a realistic assumption for control studies. As a consequence, the analytical representation of a subsea cable with three conducting layers (conductor, sheath and armour) reduces to that of an equivalent conductor. The cable can now accurately be described by the steady-state pi-model, shown in Fig. 1.

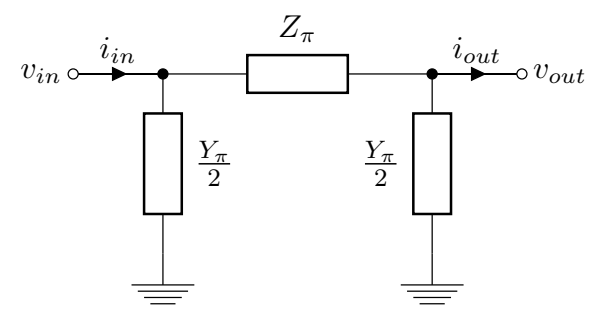

Fig. 1: Steady-state pi-model of the cable.

The series and parallel elements of this circuit are given by

$$
\begin{aligned}
& Z_{\pi}=z \ell \frac{\sinh \gamma \ell}{\gamma \ell}, \\
& Y_{\pi}=y \ell \frac{\tanh \frac{\gamma \ell}{2}}{\frac{\gamma \ell}{2}},
\end{aligned}
$$

in which $z$ and $y$ are the cable impedance and admittance per unit length, respectively

$$
\begin{aligned}
& z(\omega)=r(\omega)+\jmath \omega l(\omega), \\
& y(\omega)=g(\omega)+\jmath \omega c(\omega),
\end{aligned}
$$

and $\gamma$ the propagation constant defined as

$$
\gamma=\sqrt{z y}
$$

The frequency dependence of the conductance $g$ and the capacitance $c$ is normally omitted for electromagnetic transient (EMT) models of power cables [5], simplifying Equation (4) to

$$
y=g+\jmath \omega c .
$$

On the contrary, omitting the frequency dependence of the resistance $r$ and inductance $l$ is much more severe, as discussed in the remainder of the paper. Fig. 2 shows the frequency dependence of $r$ and $l$ for a $320 \mathrm{kV}$ XLPE cable for VSC HVDC transmission with data from [6]. Fig. 3 shows the impedance for a $300 \mathrm{~km}$ long cable with these parameters, using the analytical formulation from Equations (1)-(6), with the cable shortcircuited at one end.

The analytical equivalent pi-model can be used for frequencydependent steady-state analysis, such as harmonic studies. However, the frequency dependence of the parallel and series elements hampers a straightforward extension to a state-space model.
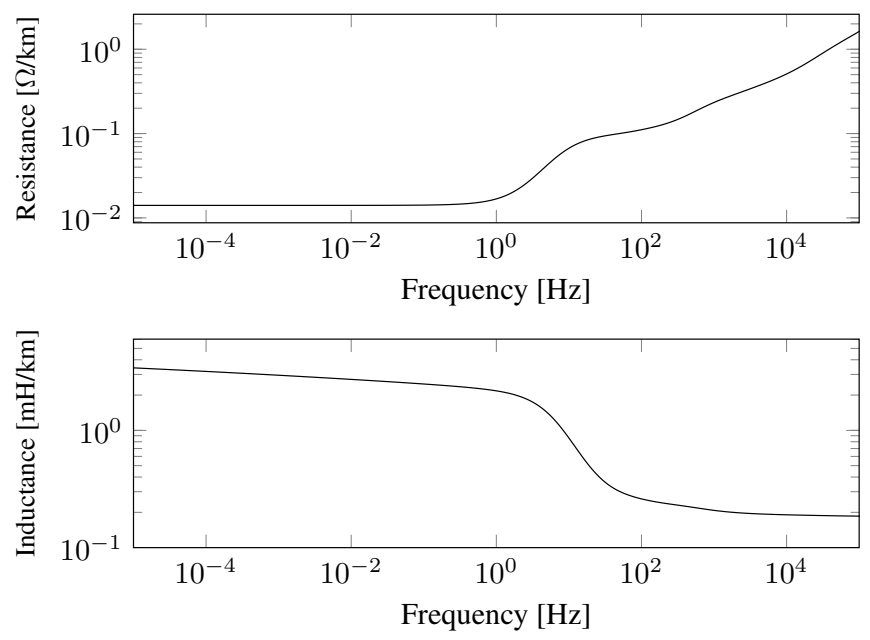

Fig. 2: Frequency dependence of $r$ and $l$.
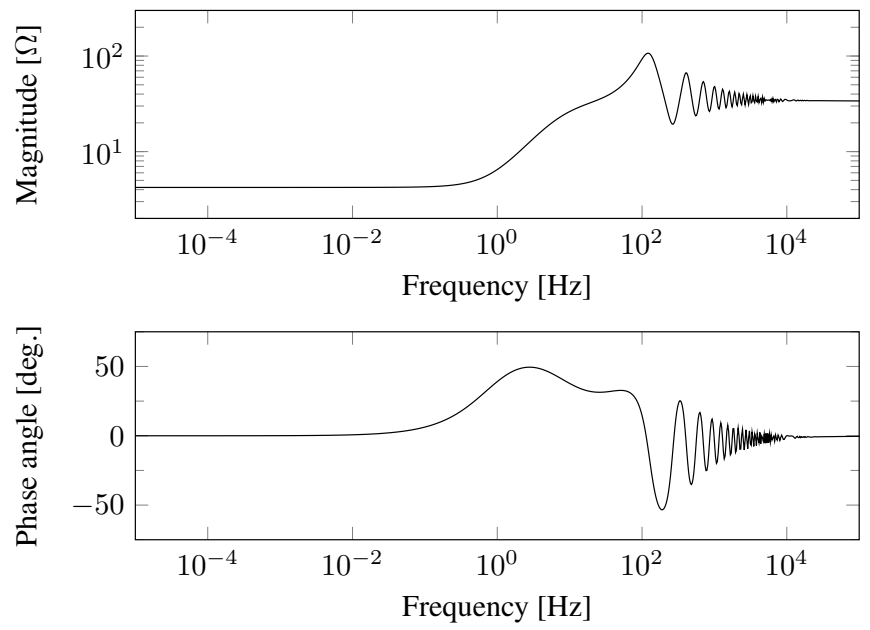

Fig. 3: Cable impedance - analytical model.

\subsection{Constant parameter approximation}

A common approach to represent the cable in a state-space model, starts with the assumption that the frequency dependence of all parameters can be omitted, hence also simplifying Equation (3) to

$$
z=r+\jmath \omega l .
$$

It is important to stress that the resulting model still relies on the analytical formulation from Equations (1)-(2), but with the constant frequency approximation from Equations (6)-(7).

Fig. 4 shows the effect of removing the frequency dependence of $r$ and $l$. In this example, the values of these parameters have been chosen to obtain an optimal fitting over the frequency range $[1 \mathrm{e}-6 . . .1 \mathrm{e} 6] \mathrm{Hz}$. The details of this fitting process are explained in the next section. In practice, the fitting process results in selecting the data for $r$ and $l$ that are relatively close to values in the low frequency range.

Alternatively, it is possible to use the values of $r$ and $l$ in Equa- 

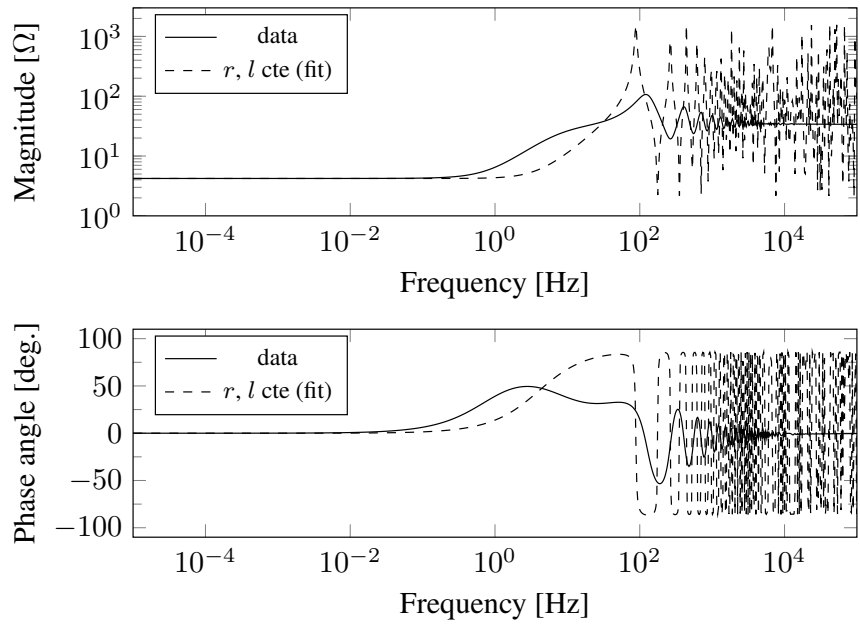

Fig. 4: Cable impedance - constant parameter approximation, fitted data.
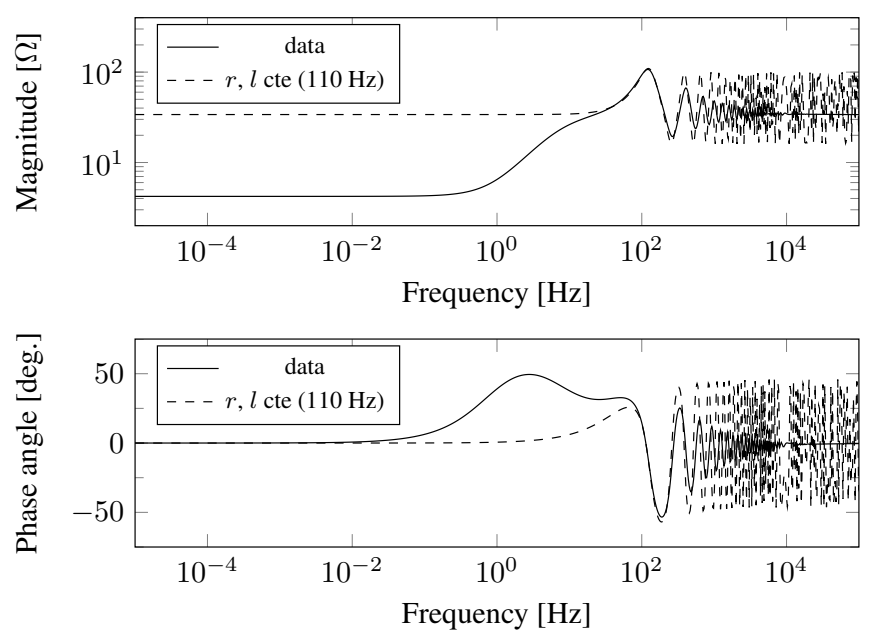

Fig. 5: Cable impedance - constant parameter approximation, data at $\mathrm{f}=110 \mathrm{~Hz}$.

tion (7) at a given frequency. Fig. 5 shows the result for data selected in the vicinity of the first resonance frequency, i.e. at $\mathrm{f}=110 \mathrm{~Hz}$. The impedance plot shows that this gives an overall better result in the vicinity of the resonance peak, but it compromises the impedance value at low frequencies, yielding amongst other different system losses.

\subsection{Standard cascaded pi-model}

As the model from the previous section still relies on the analytical formulation from Equations (1)-(2), an approximation is needed to represent it in a state-space model. A common approach is to use (cascaded) pi-sections. The approximation stems in the fact that for short lines, the hyperbolic correction factors in Equations (1)-(2) approach 1.0, resulting in a series impedance $Z \ell$. For longer lines, these correction factors can be approximated by cascading multiple pi-sections in series. This approach is taken in [7] to present a state-space model for transmission lines and is a commonly used approach to model HVDC cables for small-signal stability studies. Fig. 6 shows the approximation of the cable with respectively 1 and 10 cascaded pi-equivalents, using the constant $r$ and $l$ values from Section 2.2.
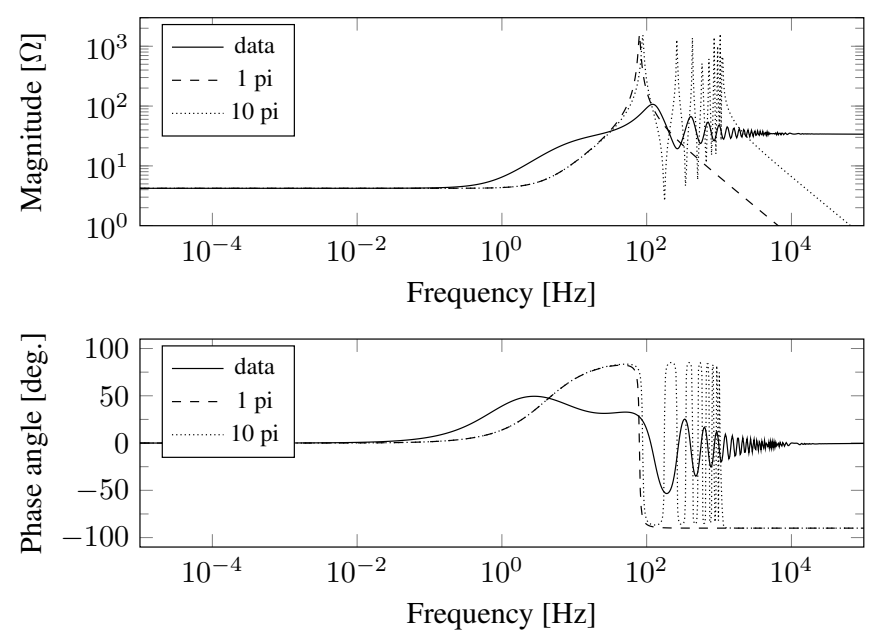

Fig. 6: Cable impedance - nominal pi-equivalent approximation.

What is clear from this picture, is that by cascading several branches, the cable response closer and closer resembles the behaviour of the analytical equivalent pi-model with constant parameters, in this particular case the model from Fig. 4. As a consequence, this does not necessarily results in a good approximation of the actual cable impedance (Fig. 3).

\section{Frequency-dependent cascaded pi-model}

What is clear from the previous section, is that increasing the number of pi-sections results in a better approximation of the hyperbolic correction factors from Equations (1)-(2). However, it is important to note that it does not necessarily results in a good approximation of the actual behaviour of the cable since it does not allow to take into account the frequency dependency of the distributed parameters.

To overcome these drawbacks, the idea of modelling the cable by means of parallel series $R L$-branches from [2] has been used. In [3], this idea has been explored to include transmission lines in a state-space model. To some extent, the method presented in this paper shows similarities to the approach taken in [1], which involved modelling the cable screen while including a coupled inductor. However, the proposed method is different since it assumes the screen to be at ground potential and it is more general in the sense that the approach can be extended to an arbitrary number of branches, thereby improving the fitting in the frequency domain. Furthermore, the approach can also be applied for other line or cable configurations.

The modelling approach consists of two subsequent steps. First, the frequency dependence of the series elements are fit- 


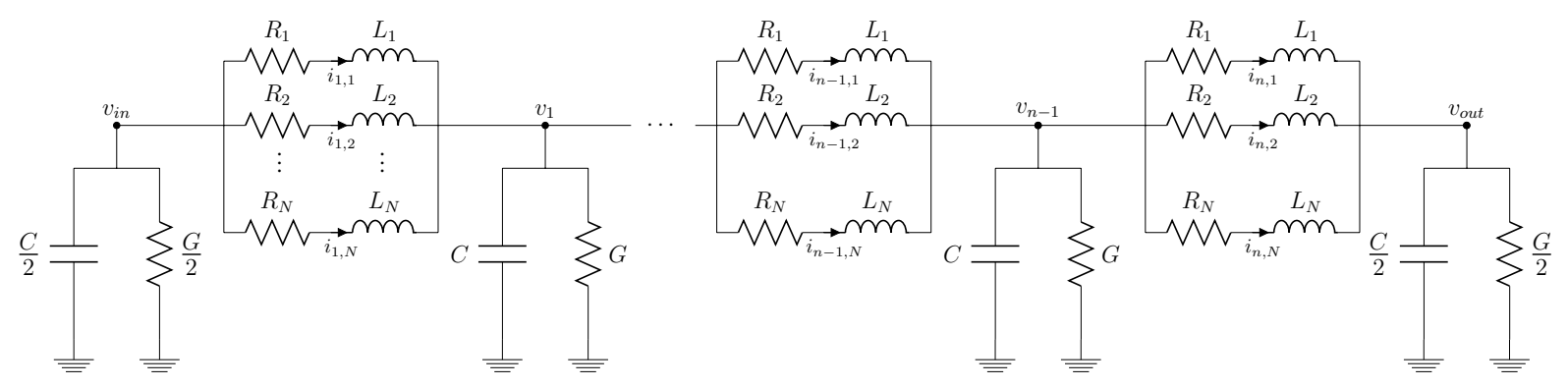

Fig. 7: Equivalent pi-model with parallel series branches.

ted with parallel branches. Second, the hyperbolic correction factors from Equations (1)-(2) are approximated with multiple pi-sections. The result is the model from Fig. 7. The elements of the pi-equivalent scheme are given by

$$
\begin{aligned}
R_{i} & =r_{i} \ell_{\pi}, \\
L_{i} & =l_{i} \ell_{\pi}, \\
G & =c \ell_{\pi}, \\
C & =g \ell_{\pi},
\end{aligned}
$$

in which $\ell_{\pi}=\ell / n$ is the length of a pi-section and $n$ represents the number of pi-sections.

\subsection{Vector fitting of series impedance elements}

The goal of the first step, the vector fitting, is to provide an adequate description in the frequency domain by means of a rational approximation of order $N$. The problem takes the general form of a sum of partial fractions [4]

$$
f(s)=\sum_{i=1}^{N} \frac{c_{i}}{s-a_{i}}+d+s e .
$$

Considering $N$ parallel branches with a resistance $r_{i}$ and inductance $l_{i}$ as in Fig. 8, the series admittance of the cable, $y_{s}(s)=[r(s)+s l(s)]^{-1}$, is in fact approximated as

$$
y_{s}(s) \approx \sum_{i=1}^{N} \frac{1}{l_{i} s+r_{i}} .
$$

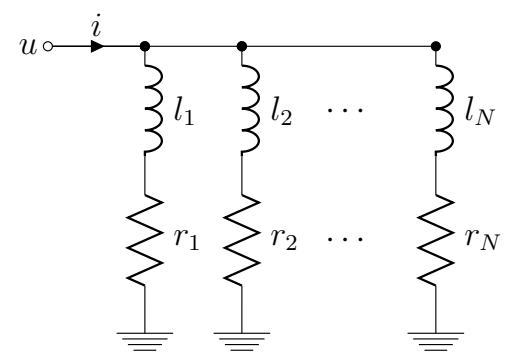

Fig. 8: Approximate representation of the series element.
A comparison of Equation (12) and Equation (13) shows that this model naturally leads to a simplified version of the rational formulation from Equation (12) with $d=0, e=0$ and

$$
\begin{aligned}
l_{i} & =c_{i}^{-1}, \\
r_{i} & =-a_{i} c_{i}^{-1},
\end{aligned}
$$

The DC resistance per unit length of the equivalent cable model is thus given by

$$
r_{d c}=\frac{1}{\sum_{i=1}^{N}-a_{i}^{-1} c_{i}} .
$$

Fig. 9 shows the approximation of the series impedance using 3 parallel branches. It is clear that the fitting of the series im-
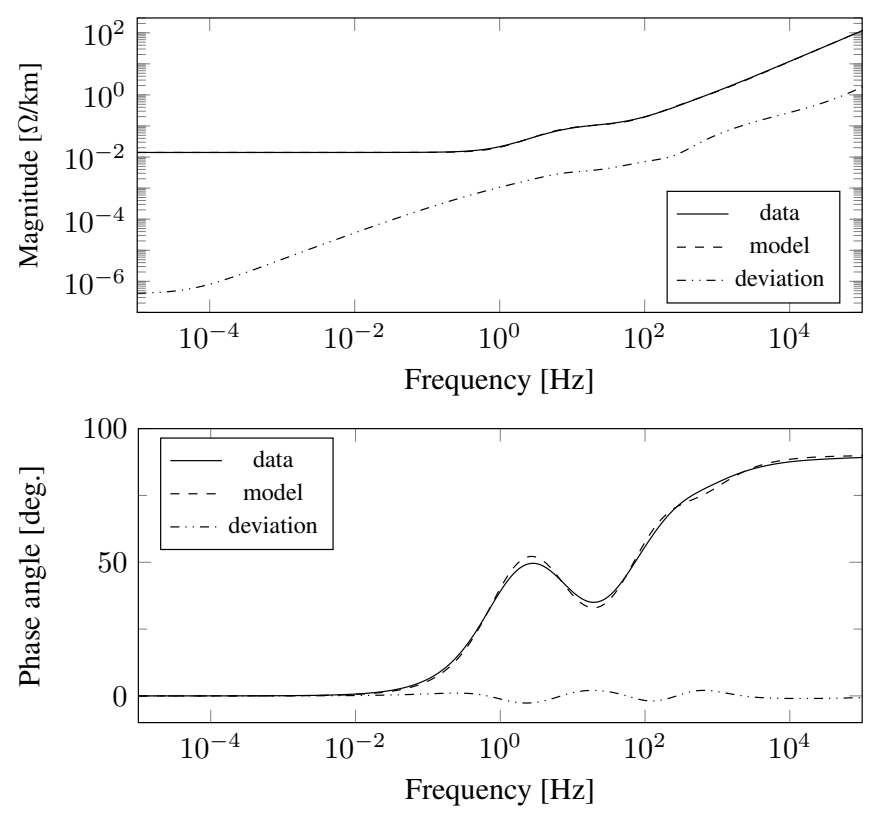

Fig. 9: Series impedance $Z$ - approximation with three parallel branches.

pedance data to parallel branches thus provides a valid means to take into account the frequency-dependence of the line parameters. 


\subsection{Cascading pi-sections}

The hyperbolic correction factors in Equations (1)-(2) can now be taken into account by using cascaded pi-equivalents with parallel series branches. The number of pi-sections depends on the modelling needs and consequentially on the bandwidth of the cable model aimed at in the state-space representation. This is illustrated in Fig. 10, which shows the results of using 1, 5 and 10 pi-sections for a cable model with 3 parallel branches.
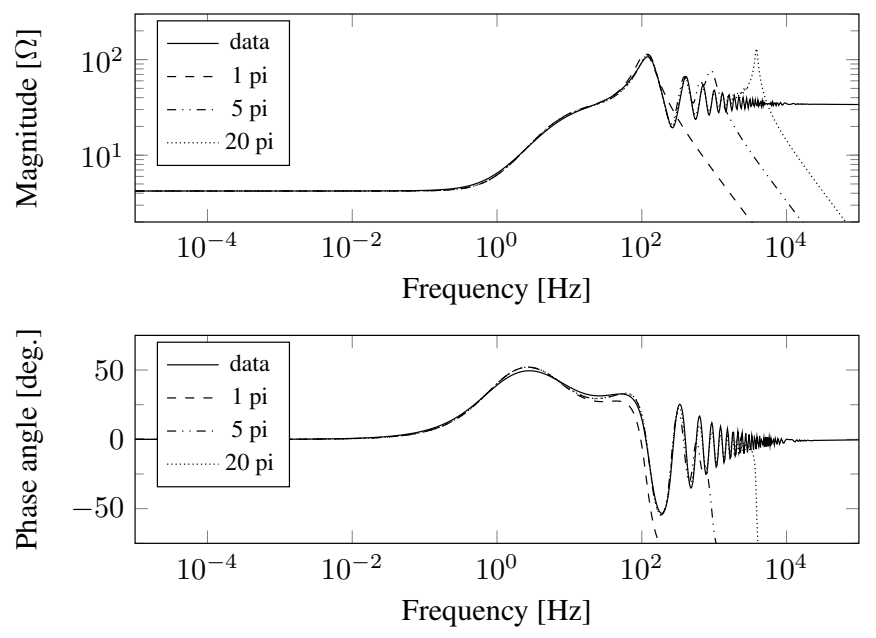

Fig. 10: Cable impedance - pi-equivalent with 3 parallel branches approximation.

From the picture, it is clear that the method as such does not pose any theoretical restrictions on the level of detail that can be represented in the model and that it can be used to accurately represent the cable in a state-space representation. The level of complexity however, increases with the number of piequivalents and the number of parallel branches.

\section{System modelling}

In order to analyse the extent to which the converter dynamics interact in the system, the different components in the HVDC system are first modelled as independent linear time-invariant subsystems. Each subsystem is modeled as a linearised system

$$
\dot{\boldsymbol{x}}=\mathrm{A} \boldsymbol{x}+\mathrm{B} \boldsymbol{u} ; \boldsymbol{x}(0)=\boldsymbol{x}_{\mathbf{0}}
$$

with $\boldsymbol{x} \in \mathbb{R}^{n}$ the state vector, $\boldsymbol{u} \in \mathbb{R}^{m}$ the input vector and $\mathrm{A} \in \mathbb{R}^{n \times n}, \mathrm{~B} \in \mathbb{R}^{n \times m}$ the known coefficient matrices that can be a function of the steady-state linearisation around $\boldsymbol{x}_{0} \in \mathbb{R}^{n}$.

The main focus of this section is on the modelling of the cable, and more specifically the inclusion of multiple pi-sections with parallel branches in a state-space modelling approach.

\subsection{Converter state-space representation}

The converter and its control loops, assuming an averaged model of the converter, can be derived in a the form of Equation (17).
The converter model used in this study is based on the model described in [8]. It is based on an averaged model of a 2level converter, including filter bus dynamics. The connection to the grid is taken into account using a complex impedance, which represents the combination of the transformer and the grid Thevenin impedance.

A phase-locked loop (PLL) is used to synchronise the $d q$ reference frame to the voltage at the filter bus. Other control loops include decoupled inner current controllers, active power or DC voltage control. Both can be extended with an outer droop control loop, resulting in two different implementations. Further features of the model include active damping components to prevent filter bus voltage oscillations from entering the control loops. Other dynamics are related to the low pass filtering of the measurements of the current, DC voltage and active power. Outer control loops for the reactive power have been left out of the study.

Fig. 11 indicates how the converter is modelled separately as a building block for the state-space representation. The current at the DC side is considered as an input to the model, as are the AC voltage source and the references for the controllers. The result is an 18th-order model, in which the DC converter voltage appears as the state variable that needs to be linked with the other components in the system. The steady-state conditions can be calculated independently for this building block by assuming a certain input vector.

\subsection{Cable state-space representation}

The model from Fig. 7 can be directly written in the state-space form from Equation (17). However, attention needs to be paid to the integration with the converter models. In the previous section, it was discussed how the converter DC voltage is a state variable. This implies that, when coupling the cable with the converter model from Fig. 11, the first parallel pi-elements (i.e. $G / 2$ and $C / 2$ Fig. 7) need to be treated as a part of the converter instead, by adding $C / 2$ to the converter capacitance. With this, the cable can be written as as state-space model with input vector $\boldsymbol{u}$ given by

$$
\boldsymbol{u}=\left[\begin{array}{c}
v_{\text {in }} \\
v_{\text {out }}
\end{array}\right] .
$$

The vector $\boldsymbol{x}$ containing the cable state variables and the matrices A and B are given by Equations (19)-(21) (see the top of next page) for the general case of a cable with $n$ pi-equivalents and $N$ parallel branches. The first and last subblock differ as the voltages in the beginning and at the end of the cable are treated as inputs to the model (see B in Equation (21)). In case only one pi-equivalent is used, matrices $A$ and B respectively simplify to an $N \times N$ and an $N \times 2$ matrix, with vector $\boldsymbol{x}$ only retaining the $N$ currents as state variables.

\subsection{Combined system state-space model}

With all different subsystems described in the form of Equation (17), a state-space model of the entire system is assembled. 


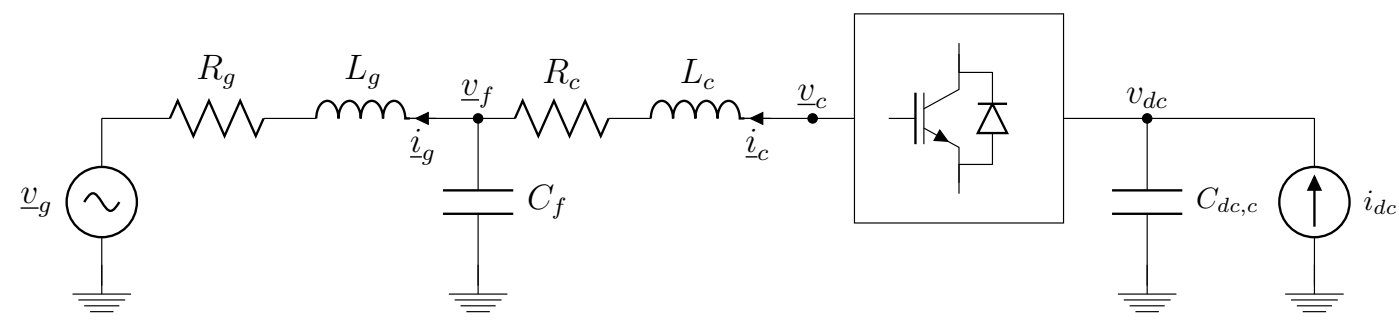

Fig. 11: Converter state-space model.

$$
\begin{aligned}
& \boldsymbol{x}=\left[\begin{array}{lllllllllllll}
i_{1,1} & \cdots & i_{1, N} & v_{1} & i_{2,1} & \cdots & i_{2, N} & v_{2} & \cdots & v_{n-1} & i_{n, 1} & \cdots & i_{n, N}
\end{array}\right]^{T}, \\
& {\left[\begin{array}{ccccccc}
\frac{R_{1}}{L_{1}} & & & -\frac{1}{L_{1}} & & & \\
& \ddots & & \vdots & & & \\
& & \frac{R_{N}}{L_{N}} & -\frac{1}{L_{N}} & & & \\
\frac{1}{C} & \ldots & \frac{1}{C} & -\frac{G}{C} & -\frac{1}{C} & \ldots & -\frac{1}{C}
\end{array}\right.} \\
& \frac{1}{L_{1}} \quad \frac{R_{1}}{L_{1}} \quad-\frac{1}{L_{1}} \\
& \vdots \quad \ddots \quad \vdots \\
& \frac{1}{L_{N}} \quad \frac{R_{N}}{L_{N}} \quad-\frac{1}{L_{N}}
\end{aligned}
$$

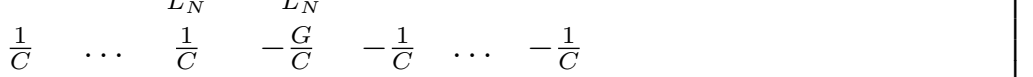

$$
\begin{aligned}
& \begin{array}{lllllll}
\ddots & \ddots & \ddots & & & & \\
\frac{1}{C} & \ldots & \frac{1}{C} & -\frac{G}{C} & -\frac{1}{C} & \ldots & -\frac{1}{C}
\end{array} \\
& \frac{1}{L_{1}} \quad \frac{R_{1}}{L_{1}}
\end{aligned}
$$

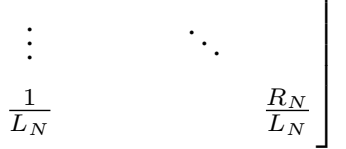

$$
\begin{aligned}
& \mathrm{B}=\left[\begin{array}{lll}
\frac{1}{L_{1}} & \cdots & \frac{1}{L_{N}} \\
& &
\end{array}\right. \\
& \left.-\frac{1}{L_{1}} \quad \cdots \quad-\frac{1}{L_{N}}\right]^{T},
\end{aligned}
$$

As an example, we consider a two-terminal HVDC system with subscripts $a, b$ and $c$ respectively referring to the first and second converter and the cable connecting the two converters.

A first step in determining the state-space model of the different components involves a linearisation for the converter equations, for which a steady-state solution for the DC system is required, taking into account the DC system losses. As a result, one obtains the state-space models of the different components

$$
\begin{aligned}
\dot{x}_{\boldsymbol{a}} & =\mathrm{A}_{\mathrm{a}} \boldsymbol{x}_{\boldsymbol{a}}+\mathrm{B}_{\mathrm{a}} \boldsymbol{u}_{\boldsymbol{a}}, \\
\dot{x}_{\boldsymbol{b}} & =\mathrm{A}_{\mathrm{b}} \boldsymbol{x}_{\boldsymbol{b}}+\mathrm{B}_{\mathrm{b}} \boldsymbol{u}_{\boldsymbol{b}}, \\
\dot{x}_{\boldsymbol{c}} & =\mathrm{A}_{\mathrm{c}} \boldsymbol{x}_{\boldsymbol{c}}+\mathrm{B}_{\mathrm{c}} \boldsymbol{u}_{\boldsymbol{c}},
\end{aligned}
$$

with $\boldsymbol{x}_{\boldsymbol{a}} \in \mathbb{R}^{n_{a}}, \boldsymbol{x}_{\boldsymbol{b}} \in \mathbb{R}^{n_{b}}, \boldsymbol{x}_{\boldsymbol{c}} \in \mathbb{R}^{n_{c}}$ the components' state variables, $\boldsymbol{u}_{\boldsymbol{a}} \in \mathbb{R}^{m_{a}}, \boldsymbol{u}_{\boldsymbol{b}} \in \mathbb{R}^{m_{b}}, \boldsymbol{u}_{\boldsymbol{c}} \in \mathbb{R}^{m_{c}}$ their input variables, $\mathrm{A}_{\mathrm{a}} \in \mathbb{R}^{n_{a} \times n_{a}}, \mathrm{~A}_{\mathrm{b}} \in \mathbb{R}^{n_{b} \times n_{b}}, \mathrm{~A}_{c} \in \mathbb{R}^{n_{c} \times n_{c}}$ their state-space matrices and $\mathrm{B}_{\mathrm{a}} \in \mathbb{R}^{n_{a} \times m_{a}}, \mathrm{~B}_{\mathrm{b}} \in \mathbb{R}^{n_{b} \times m_{b}}, \mathrm{~B}_{\mathrm{c}} \in \mathbb{R}^{n_{c} \times m_{c}}$ their input matrices.

The overall system matrix $A_{t}$ can be assembled by accounting for the state variables of the different models that are input variables to the model of other components. More specifically, these are the DC currents at the cable ends and the converter DC voltages.

The total state-space model can be written as

$$
\dot{\boldsymbol{x}}_{\boldsymbol{t}}=\mathrm{A}_{\mathrm{t}} \boldsymbol{x}_{\boldsymbol{t}}+\mathrm{B}_{\mathrm{t}} \boldsymbol{u}_{\boldsymbol{t}},
$$


with

$$
\begin{gathered}
x_{t}=\left[\begin{array}{l}
x_{a} \\
x_{b} \\
x_{c}
\end{array}\right], \\
u_{t}=\left[\begin{array}{l}
u_{a}^{r} \\
u_{b}^{r}
\end{array}\right] .
\end{gathered}
$$

Without overlapping states in the modelling of the components, $\boldsymbol{x}_{\boldsymbol{t}} \in \mathbb{R}^{n_{t}}$ with $n_{t}=n_{a}+n_{b}+n_{c}$. The total input vector $\boldsymbol{u}_{\boldsymbol{t}} \in \mathbb{R}^{m_{t}}$ only consists of reduced versions $\boldsymbol{u}_{\boldsymbol{a}}^{\boldsymbol{r}} \in \mathbb{R}^{m_{a}^{r}}$, $\boldsymbol{u}_{\boldsymbol{b}}^{r} \in \mathbb{R}^{m_{b}^{r}}$ of the converter input vectors $\boldsymbol{u}_{\boldsymbol{a}} \in \mathbb{R}^{m_{a}}$ and $\boldsymbol{u}_{\boldsymbol{b}} \in \mathbb{R}^{m_{b}}$, since the DC currents are no longer inputs to the model and since the inputs of the cable model have been internalised because they are state variables of the converters.

Without lack of generality, let $k$ denote the index of the DC voltage $v_{d c}$ in the state variable vectors of the converters $\boldsymbol{x}_{\boldsymbol{a}}$ and $\boldsymbol{x}_{\boldsymbol{b}}$ such that $x_{a_{k}}=v_{d c, a}$ and $x_{b_{k}}=v_{d c, b}$ and let $k_{a}$ and $k_{b}$ denote their indices in the combined state variables vector $\boldsymbol{x}_{\boldsymbol{t}}$.

For the cables, these two voltages are the only inputs to the model. When building the state-space representation of the entire system, the corresponding input matrix elements $\boldsymbol{B}_{\boldsymbol{c}_{1}}$ and $\boldsymbol{B}_{\boldsymbol{c}_{2}}$, the columns of the cable input matrix $B_{c}$, are therefore combined into the system state-space matrix $A_{t}$ as given by Equation (28).

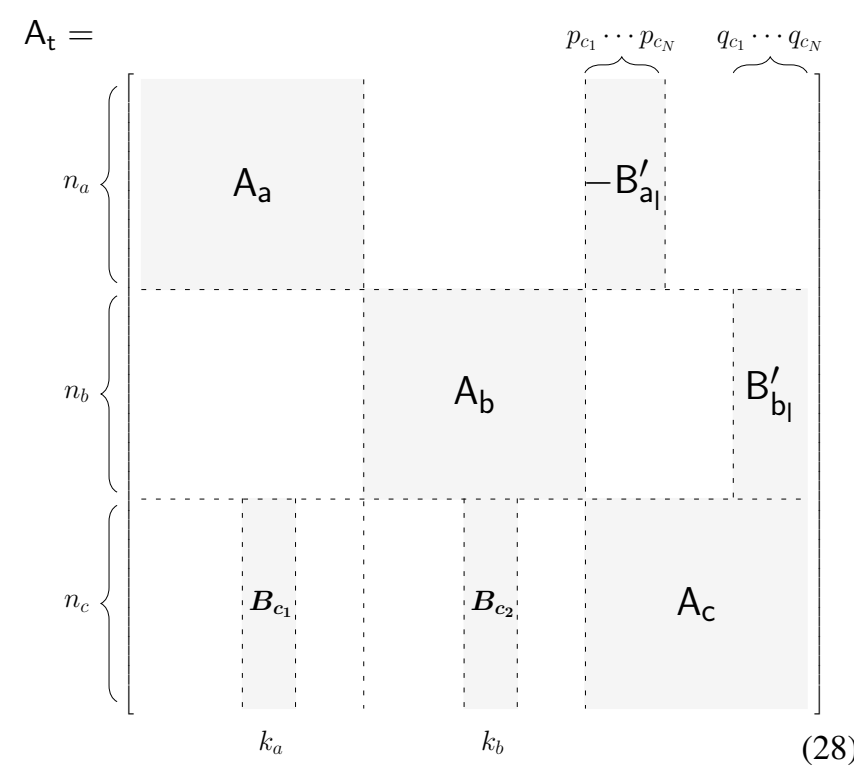

Similarly, let $l$ denote the index of the $i_{d c}$ in the input vector of these converters $\boldsymbol{u}_{\boldsymbol{a}}$ and $\boldsymbol{u}_{\boldsymbol{b}}$, such that $u_{a_{l}}=i_{d c, a}$ and $u_{b_{l}}=$ $i_{d c, b}$ and the corresponding elements in the input matrices $\mathrm{B}_{\mathrm{a}}$ and $\mathrm{B}_{\mathrm{b}}$ are given by $\boldsymbol{B}_{\boldsymbol{a}_{l}}$ and $\boldsymbol{B}_{\boldsymbol{b}_{l}}$, i.e. the $l$-th column in these input matrices.

When coupling the model of the cable to the converter, the equivalent current source $i_{d c}$ from Fig. 11 is a sum of the currents from the different parallel branches. For the converter $a$, the current is defined to flow into the cable, whereas the opposite holds for converter $b$, hence

$$
\begin{aligned}
i_{d c, a} & =-\sum_{j=1}^{N} i_{1, j}, \\
i_{d c, b} & =\sum_{j=1}^{N} i_{n, j} .
\end{aligned}
$$

Let $p_{c_{i}} \in \mathcal{I}^{\boldsymbol{i}_{1}}$ and $q_{c_{i}} \in \mathcal{I}^{\boldsymbol{i}_{\boldsymbol{n}}}$ denote the indices of the currents respectively flowing in and out of the cable. This implies that the corresponding input matrix columns $\boldsymbol{B}_{\boldsymbol{a}_{l}}$ and $\boldsymbol{B}_{\boldsymbol{b}_{l}}$ need to be included for each parallel branch current, or, assuming that all currents flowing in and out of the cable are grouped as in Equation (19), $\mathrm{B}_{\mathrm{a}_{\mathrm{l}}}^{\prime}=\boldsymbol{B}_{\boldsymbol{a}_{l}} \cdot \overrightarrow{\mathbf{1}}_{\boldsymbol{N}}^{T}$ and $\mathrm{B}_{\mathrm{b}_{\mathrm{l}}}^{\prime}=\boldsymbol{B}_{\boldsymbol{b}_{l}} \cdot \overrightarrow{\mathbf{1}}_{\boldsymbol{N}}{ }^{T}$ with $\overrightarrow{\mathbf{1}}_{\boldsymbol{N}}$ the ones vector with $\operatorname{dim}\left(\overrightarrow{\mathbf{1}}_{N}\right)=N$. Taking into account the direction of the current from Equations (29)-(30), the matrices are put into the overall state-space matrix $A_{t}$ given by Equation (28).

The elimination of the currents and the voltages as inputs to the model only preserves the converter references, and the AC system voltage as inputs to the model. With $B_{a}^{r}$ and $B_{b}^{r}$ the reduced input matrices of the converters after elimination of the elements related to the $\mathrm{DC}$ currents, the system input matrix $\mathrm{B}$ is given by Equation (31).

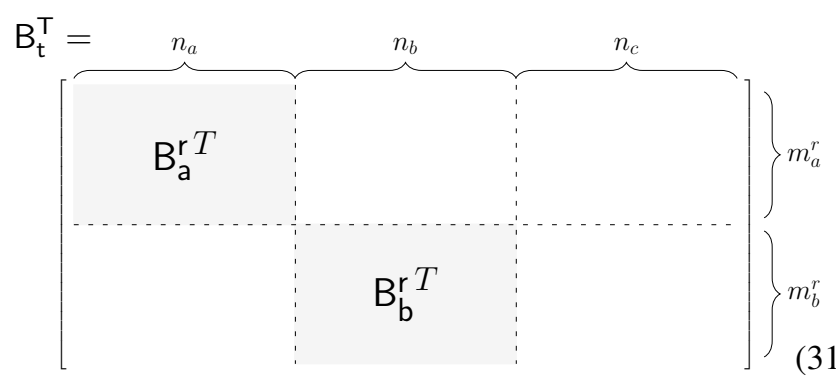

\section{Model reduction for interoperability studies}

The remainder of this work focuses on setting up guidelines for model reduction of the state-space model based on the transfer characteristics of the cable and the bandwidth of the converter control loops. Fig. 10 shows that adding more pi-sections to the model, whilst keeping the number of parallel branches constant, increases the bandwidth of the cable model.

The following procedure is therefore proposed:

Step 1 Determination of the converter model bandwidth. The maximum oscillation frequency of the converter is approximated from the eigenvalue analysis, e.g. for converter $a$

$$
\omega_{B, a}=\max _{\lambda_{a_{i}} \in \sigma\left(\mathrm{A}_{\mathrm{a}}\right)}\left|\Im\left(\lambda_{a_{i}}\right)\right|
$$

with $\lambda_{a_{i}}$ the eigenvalues and $\sigma\left(\mathrm{A}_{\mathrm{a}}\right)$ the spectrum of $\mathrm{A}_{\mathrm{a}} \cdot \omega_{B, a}$ forms an indication for the maximum modelling detail required 
for the HVDC transmission system to study converter interactions.

Step 2 Determination of the cable modelling requirements. The converter model bandwidth $\omega_{B}$ provides a first approximation for the required cable modelling requirements. As a first conservative assumption, the cable bandwidth is matched with the converter model bandwidth $\omega_{B}$, since this gives an indication for the maximum frequency at which interactions between the converters can take place. It is important to recall that the number of parallel branches determines the fitting accuracy of the series element, whereas the bandwidth of the cable is primarily determined by the number of pi-sections.

\section{Step 3 Study of converter interactions.}

With the obtained system model from Equation (25), interactions between the converters are studied. To do so, the local modes need to be separated from the interaction modes. Interacting modes can be defined as modes in which the two converters participate, which can be classified on the basis of participation factors. Let $p_{k i}$ denote the participation factor of state variable $x_{k}$ in mode $i, \boldsymbol{p}_{\boldsymbol{i}} \in \mathbb{R}^{n_{t}}$ the vector with the participation factors for all system states associated with mode $i$, and $\boldsymbol{p}_{\boldsymbol{\alpha}, i} \in \mathbb{R}^{n_{\alpha}}$ the vector for all states of subsystem $\alpha$. We now define the parameter $\eta_{\alpha i}$ as a measure for the overall participation for each subsystem $\alpha$ in mode $i$ such that

$$
\eta_{\alpha i}=\frac{\left\|\boldsymbol{p}_{\boldsymbol{\alpha}, \boldsymbol{i}}\right\|}{\left\|\boldsymbol{p}_{\boldsymbol{i}}\right\|},
$$

with $\|\cdot\|$ denoting the $L_{1}$-norm. $\eta_{a i}, \eta_{b i}$ and $\eta_{c i}$ are a measure for the degree to which the 2 converters and the cable participate in each mode. Defining a threshold $\chi$, we define an interacting mode $i$ as a mode for which both $\eta_{a i}>\chi$ and $\eta_{b i}>\chi$ resulting in a subset of interacting modes $\mathcal{S}$.

\section{Step 4 Interaction-based model reduction.}

A fourth and final step in the analysis is to reduce the system model based on the converter interactions that need to be preserved in the model. The first step in the analysis took the conservative approach that the cable model needs to be accurate over the entire range of the converters' bandwidth $\omega_{B}$. After having analysed the participation of the converter state variables in the different modes, we define a reduced bandwidth requirement as one which yields an accurate representation of the interacting mode with the highest frequency or

$$
\omega_{B, \mathcal{S}}=\max _{i \in \mathcal{S}}\left|\Im\left(\lambda_{t_{i}}\right)\right|,
$$

This overall bandwidth is defined for the entire system. One should be careful when using this number to reduce the cable bandwidth, since the corresponding eigenvalues $\lambda_{i}$ with $i \in \mathcal{S}$ do not entirely coincide with the eigenvalues $\lambda_{c, i}$ of the cable state-space matrix $A_{c}$ due to the influence of the converter. However, for low values of the threshold $\chi$, it is a valid assumption that the mode determining the cable bandwidth requirements (denoted as $\lambda_{B, \mathcal{S}}$ ) only has a limited participation from the converter state variables $\boldsymbol{x}_{\boldsymbol{a}}$ and $\boldsymbol{x}_{\boldsymbol{b}}$ and thus is mainly associated with the cable state variables $x_{\boldsymbol{c}}$. Consequentially, the position of $\lambda_{B, \mathcal{S}}$ will largely coincide with a corresponding eigenvalue of the cable model. Hence, $\omega_{B, \mathcal{S}}$ can be used as the new bandwidth requirements for the cable model.

Similarly, the converter model can now be reduced by emphasising the modes that have a participation from both converters when reducing the overall order of the system. In this paper, the focus is on the model reduction of the cable.

\section{Simulation results}

In order to validate the proposed methodology, simulations are carried out for a $\pm 320 \mathrm{kV}, 900 \mathrm{MW}$ two-terminal VSC HVDC link with a length of $300 \mathrm{~km}$. Converters $a$ and $b$ are respectively set to constant voltage and constant power control. Fig. 12a shows the eigenvalues of $\mathrm{A}_{\mathrm{a}}$ (converter $a$ ), the DC voltage controlling converter. The bandwidth of the converter models $f_{B, a}$ and $f_{B, b}$ from Equation (32) is in this case equal to $581 \mathrm{~Hz}$.The states that are mainly associated with these modes are resulting from the LC circuit at the AC side and hence, little impact on their position can be expected when connecting the cable. The conservative assumption that the model for the cable needs to be accurate until this frequency, results in a cable model with 9 parallel branches and 15 pisections. Doing so, the impedance angle deviation is limited to less than $0.5^{\circ}$ in the low frequency region and is limited to $2^{\circ}$ at $f_{B, a}$. Similarly, the impedance magnitude deviation is limited to $7 \%$ at $f_{B, a}$. Fig. $12 \mathrm{~b}$ shows the resulting eigenvalues for the combined system. The eigenvalues with real parts lower than -1000 and imaginary parts over 10000 have not been depicted in this figure as these modes are largely related to internal cable states. Fig. 12c shows eigenvalues that are the result of the interaction study after defining $\eta_{\alpha i}$ for the different components $\alpha$ for mode $i$. With a threshold $\chi=5 \%, 7$ interacting poles are identified. Fig. 13 shows the overall participation of the different components in these modes. It is clear that the largest participation is visible for modes $\mathrm{F}$ and $\mathrm{G}$. The more we move to the left in the complex plane, the smaller the participation from the converters becomes. With this, we now reduce the required cable bandwidth to $297 \mathrm{~Hz}$, corresponding to the frequency of the highest interacting mode of interest. It was found that a model with 5 parallel branches and 5 pi-equivalents provides a good compromise to still accurately represent the eigenvalues of interest. Fig. 14 shows the effect of lowering the number of parallel branches and pi-sections. It is clear from this picture that keeping the number of parallel branches to 9 whilst lowering the number of pi-sections, leaves the poles $\mathrm{C}$ to $\mathrm{G}$ largely unaltered and only impacts $\mathrm{A}$ and $\mathrm{B}$, the poles with the highest imaginary parts. Also lowering the number of parallel branches to 5 , also impacts the poles $\mathrm{C}$ and $\mathrm{D}$. The number of parallel branches can be lowered to 3 , which forms a lower limit to still represent the complex conjugate poles $\mathrm{A}$ and $\mathrm{B}$. It is clear from this picture that including a number of parallel branches whilst only using one pi-equivalent still allows an accurate representation of the poles C and D. It is also clear 


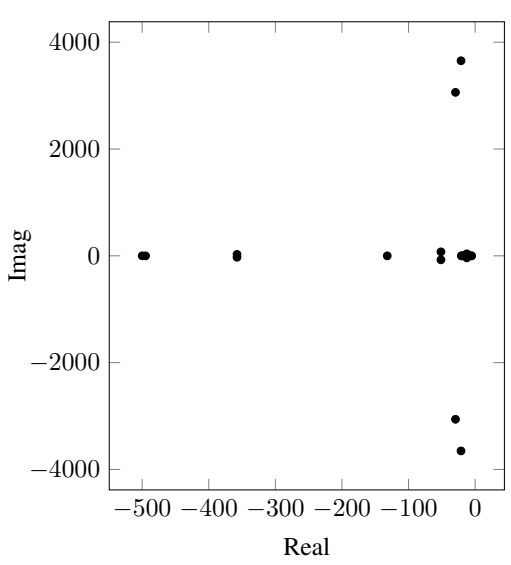

(a) Eigenvalues $\mathrm{A}_{\mathrm{a}}$ (converter $a$ )

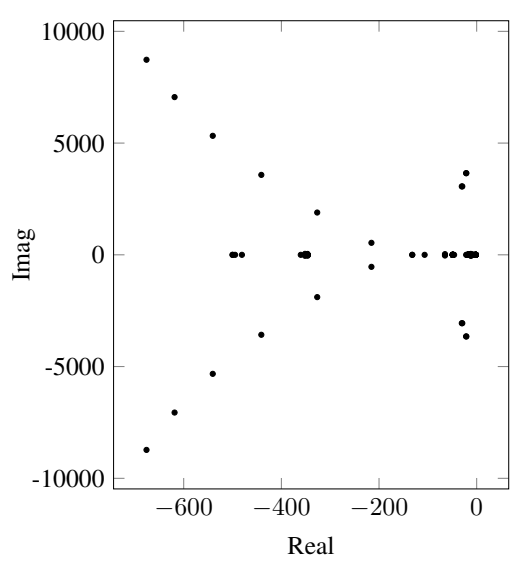

(b) Eigenvalues $A_{t}$ (system)

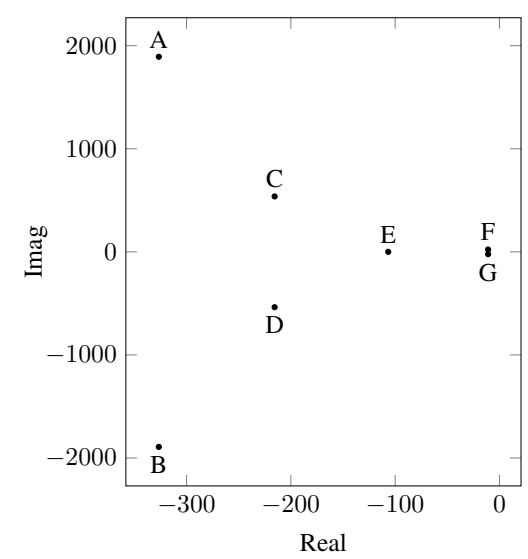

(c) Eigenvalues $\mathrm{A}_{a}$ with $\eta_{a}>\chi$ and $\eta_{b}>\chi$

Fig. 12: Eigenvalues of the converter matrix $A_{a}$, the system matrix $A_{t}$ and eigenvalues with interaction.

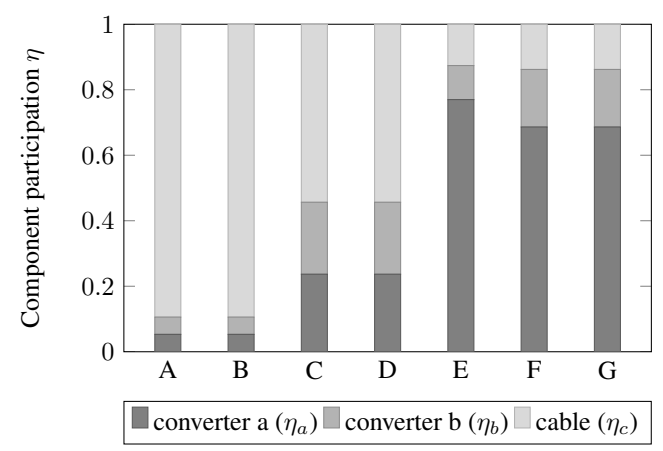

Fig. 13: Participation of component in modes with interactions between the converters.

from Fig. 14 (see detail) that all models accurately represent the interacting poles with the lowest damping, with the lowest accuracy for the simplest models with only three parallel branches. These models are slightly conservative with respect to the damping of modes $\mathrm{C}, \mathrm{D}, \mathrm{F}$ and $\mathrm{G}$ and can in this case still be used for stability assessment.

Finally, Fig. 15 shows the effect of the simplifications that are commonly encountered in state-space representation and their effect on the placements of the poles involved in the interacting modes (i.e.satisfying $\eta_{a}>\chi$ and $\eta_{b}>\chi$ ). It is clear from this figure that the simplifications based on using one parallel branch (and either one or multiple pi-sections) give misleading impressions about the relative stability of the cable modes (denoted A,B, C and D in Fig.12c) : the corresponding eigenvalues not only appear at different frequencies, but are also poorly damped. The fitted values (hence approximating the analytical representation from Fig. 4) lead to the least damped modes. The representation of the lower order cable modes (see detail) is also less accurate than the representations from Fig. 14, and even than the model using one pi-section and 3 parallel branches. Also the representation of the real pole ( $\mathrm{E}$ in Fig.12c) is less accurate. It can be noted that the simplest

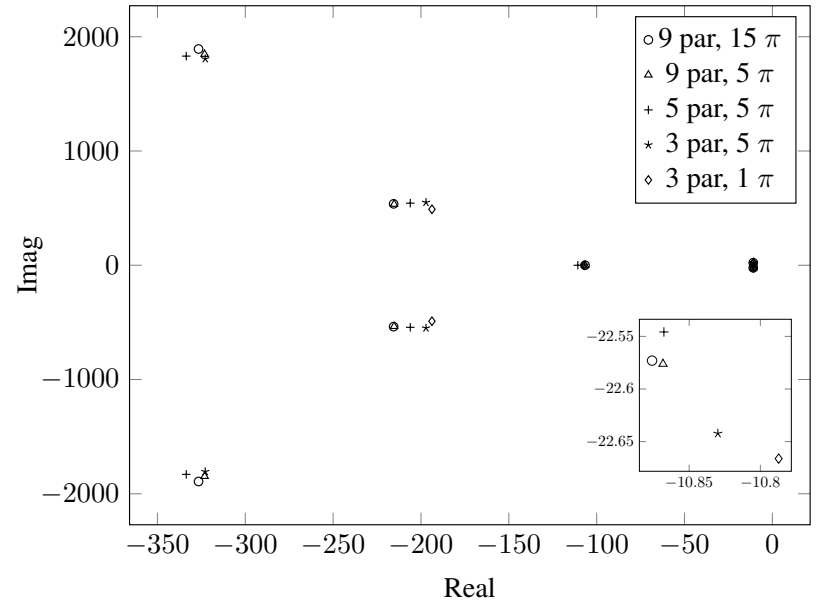

Fig. 14: Cable model reduction preserving interacting modes.

representation, only using the resistive cable value and leaving out the current as a state variable, results in similar values for modes $\mathrm{E}, \mathrm{F}$ and $\mathrm{G}$, but does not include the wrongly predicted cable modes resulting from the classical pi-equivalent models. Fig. 16 shows the comparison of the component participation $\eta$ in the interacting modes, for the reference case, as well as for the simplified cable representations without parallel branches, but with 5 pi-equivalents. Even though this figure indicates that the component participation in the different modes is similar, one should keep in mind that the location of these poles is significantly different, leading to a wrong assessment of the dynamic response and stability margin of the system.

\section{Conclusion}

In this paper, different state-space models for HVDC cables have been analysed with the purpose of accurately addressing converter interactions in HVDC systems. The models generally encountered in literature using cascaded pi-equivalents 


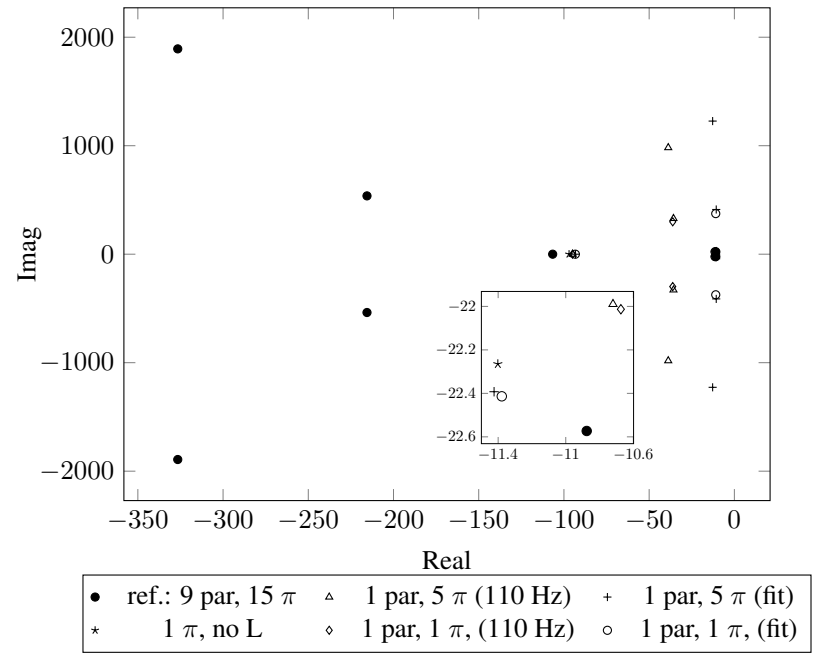

Fig. 15: Simplified cable representations and their effect on the interacting system modes (reference case: 9 parallel branches, 15 pi-equivalents).

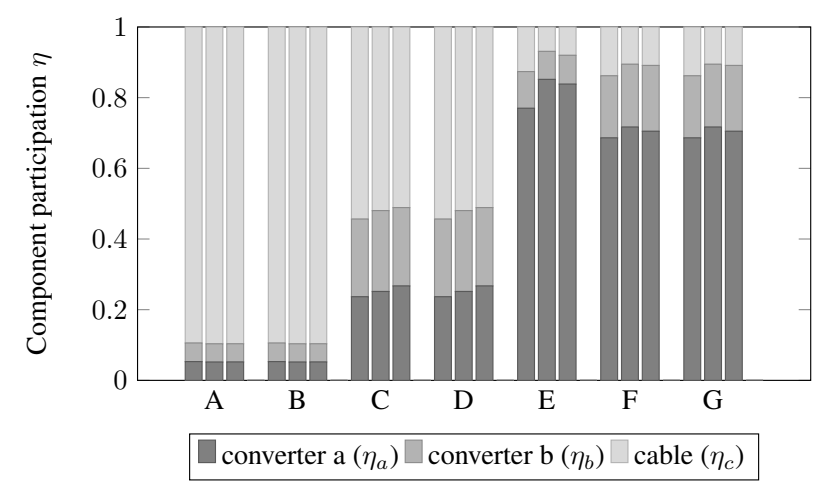

Fig. 16: Participation of components in interacting modes for simplified cable representations. Bar group legend: left: 9 par, $15 \pi$ (reference), middle: 1 par, $5 \pi(110 \mathrm{~Hz})$, right: 1 par, $5 \pi$ (fit).

result in a poor representation of the cable modes in the statespace model. This can lead to false conclusions on the dynamic response and stability margin of HVDC systems. Similarly, it is shown that, instead of using only one series branch, it can be better to leave out the cable dynamics altogether by representing the cable by its resistance. As an alternative, a model with parallel branches has been proposed, based on a vector fitting of the series elements of the cable. The model allows for an an accurate representation of the cable in the frequency domain and provides a detailed state-space representation. A proposed model reduction for the cable shows that it is possible to accurately model interacting converter modes with the model and leads to the general conclusion that the cable should preferably be modelled by a combination of parallel branches and pi-sections. In case a very simple model is sought for, it is better to model the cable using only parallel branches instead of merely cascading pi-sections.

\section{Acknowledgements}

The authors would like to thank B. Gustavsen (SINTEF) for his assistance with the vector fitting tool and W. Leterme (KU Leuven) for providing the cable data.

Jef Beerten is funded by a postdoctoral research grant from the Research Foundation - Flanders (FWO).

The work of SINTEF Energy Research in this paper was supported by the project "Protection and Fault Handling in Offshore HVDC Grids - ProOfGrids," financed by the Norwegian Research Council together with industry partners EDF, National Grid, Siemens, Statkraft, Statnett, Statoil and NVE.

\section{References}

[1] P. Rault, "Dynamic modeling and control of multiterminal HVDC grids," Ph.D. dissertation, Ecole Centrale de Lille, Mar. 2014.

[2] A. Semlyen and A. Deri, "Time domain modelling of frequency dependent three-phase transmission line impedance," IEEE Trans. Power App. Syst., vol. PAS-104, no. 6, pp. 1549-1555, Jun. 1985.

[3] N. Garcia and E. Acha, "Transmission line model with frequency dependency and propagation effects: A model order reduction and state-space approach," in Proc. PES General Meeting 2008, Pittsburgh, USA, Jul., 20-24 2008, 7 pages.

[4] B. Gustavsen and A. Semlyen, "Rational approximation of frequency domain responses by vector fitting," IEEE Trans. Power Del., vol. 14, no. 3, pp. 1052-1061, Jul. 1999.

[5] B. Gustavsen, T. Noda, J. Naredo, F. Uribe, and J. Martinez-Velasco, Power System Transients: Parameter Determination. Taylor and Francis Group, 2010, ch. 3, pp. 137-175.

[6] W. Leterme, N. Ahmed, J. Beerten, L. Ängquist, D. Van Hertem, and S. Norrga, "A new HVDC grid test system for HVDC grid dynamics and protection studies in EMT-type software," in Proc. IET ACDC 2015, Birmingham, UK, Feb., 10-12 2015, 7 pages.

[7] J. Rosendo Macías, A. Gómez Expósito, and A. Bachiller Soler, "A comparison of techniques for state-space transient analysis of transmission lines," IEEE Trans. Power Del., vol. 20, no. 2, pp. 894-903, Apr. 2005.

[8] S. D'Arco, J. A. Suul, and M. Molinas, "Implementation and analysis of a control scheme for damping of oscillations in VSC-based HVDC grids," in Proc. PEMC 2014, Antalya, Turkey, Sep., 21-24 2014, 8 pages. 